\title{
Ex-vivo Sensitivity of Plasmodium falciparum to Common Anti-malarial Drugs: The Case of Kéniéroba, a Malaria Endemic Village in Mali
}

\author{
Karim Traoré ${ }^{\text {} ~ S e i d i n a ~ A . ~ S . ~ D i a k i t e ́ ~}{ }^{1} \cdot$ Sekou Bah ${ }^{2}$. Drissa S. Konaté ${ }^{1}$. Djeneba Dabitao ${ }^{1}$ Ibrahim Sanogo ${ }^{1}$. \\ Modibo Sangaré ${ }^{\text {- Souleymane Dama }}{ }^{1}$. Bourama Keita ${ }^{1}$ Mory Doumbouya ${ }^{1}$ Merepen A. Guindo ${ }^{1}$. \\ Seydou Doumbia ${ }^{1} \cdot$ Mahamadou Diakité $^{1}$
}

Published online: 18 June 2020

(c) The Author(s) 2020

\begin{abstract}
Background In 2006, the National Malaria Control Program in Mali recommended artemisinin-based combination therapy as the first-line treatment for uncomplicated malaria. Since the introduction of artemisinin-based combination therapy, few reports are available on the level of resistance of Plasmodium falciparum to the most common anti-malarial drugs in Mali. Methods From 2016 to 2017, we assessed the ex-vivo drug sensitivity of $P$. falciparum isolates in Kéniéroba, a village located in a rural area of southern Mali. We collected P. falciparum isolates from malaria-infected children living in Kéniéroba. The isolates were tested for ex-vivo sensitivity to commonly used anti-malarial drugs, namely chloroquine, quinine, amodiaquine, mefloquine, lumefantrine, dihydroartermisinin, and piperaquine. We used the $50 \%$ inhibitory concentration determination method, which is based on the incorporation of SYBR ${ }^{\circledR}$ Green into the parasite's genetic material.

Results Plasmodium falciparum isolates were found to have a reduced ex-vivo sensitivity to quinine (25.7\%), chloroquine (12.2\%), amodiaquine (2.7\%), and mefloquine (1.3\%). In contrast, the isolates were $100 \%$ sensitive to lumefantrine, dihydroartermisinin, and piperaquine. A statistically significant correlation was found between $50 \%$ inhibitory concentration values of quinine and amodiaquine $(r=0.80 ; p<0.0001)$.

Conclusions Plasmodium falciparum isolates were highly sensitive to dihydroartermisinin, lumefantrine, and piperaquine and less sensitive to amodiaquine $(n=2)$, mefloquine $(n=1)$, and quinine $(n=19)$. Therefore, our data support the previously reported increasing trend in chloroquine sensitivity in Mali.
\end{abstract}

\section{Background}

The resistance of Plasmodium falciparum to common antimalarial drugs represents a serious hurdle for malaria control in endemic countries [1]. Plasmodium falciparum has developed resistance to low-cost and well-tolerated anti-malarial drugs such as chloroquine (CQ), amodiaquine (AQ), antifolates, and mefloquine (MQ) [2-5]. This has led the World Health Organization and National Malaria Control Program (NMCP) to recommend artemisinin-based combination

Karim Traoré

ktraore@icermali.org

1 Malaria Research and Training Center, Mali International Center for Excellence in Research (Mali-ICER), University of Sciences, Techniques and Technologies of Bamako (USTTB), BP 1805, Bamako, Mali

2 Hospital of Point-G/University of Sciences, Techniques and Technologies of Bamako (USTTB), Bamako, Mali therapy (ACT) for malaria treatment [6]. There are two main reasons for using ACT in the clinical care of malaria. First, ACT relies on the high efficacy of artemisinin, which is a drug known to suppress disease progression in P. falciparum-infected individuals at an earlier stage of infection. Second, combining artemisinin with other anti-malarial drugs will likely delay the emergence and spread of parasites that are resistant to artemisinin. This is an important consideration, given that $P$. falciparum isolates that are resistant to artemisinin-derivative drugs have been detected in Southeast Asia [7, 8]. Specifically, mutations in the kelch (K13) gene have been associated with resistance to artemisinin in vitro in Asia [9-11]. To our knowledge, such resistance to artemisinin has not been reported in Sub-Saharan Africa yet, and ACT is still very effective in that part of the continent $[9,12]$. However, Sub-Saharan Africa remains under threat because of the widespread use of ACT and the increasing intercontinental human migrations. In addition, the circulation of sub-standard or counterfeit drugs combined with 
poor adherence of patients to antimalarial treatment may contribute to a rapid selection of resistant malaria parasites in Sub-Saharan Africa.

The Malian NMCP adopted ACT as the first-line treatment for uncomplicated malaria in 2006. Notably, artemether-lumefantrine (LUM) and AQ-artesunate are used for uncomplicated malaria treatment in Mali. In addition, three drugs namely, artesunate, artemether, and quinine (QN) were designated to be used in the clinical management of severe malaria via the intravenous route. It is therefore necessary to perform regular monitoring of antimalarial drug resistance in this setting. The ex-vivo drug susceptibility testing is one of the most efficient indirect approaches to assess the efficacy of anti-malarial drugs. Few data are available on in-vitro and ex-vivo efficacy of artemisinin alone or in association with other ACT drugs on isolates circulating in Mali. In 2017, Dama et al. reported a reduced ex-vivo susceptibility of $P$. falciparum after oral artemether-LUM treatment in Mali. This study did not include key anti-malarial drugs such as $\mathrm{CQ}, \mathrm{QN}, \mathrm{AQ}, \mathrm{MQ}$, and piperaquine (PPQ). Therefore, additional studies are needed for a comprehensive analysis of drug resistance during malaria treatment to help national and international policy makers in providing evidence-based guidance about clinical management. Here, we report ex-vivo sensitivity of $P$. falciparum isolates to seven (7) common antimalarial drugs in Kéniéroba, in southern Mali.

\section{Materials and Methods}

\subsection{Collection of Plasmodium falciparum Isolates}

From June 2016 to October 2017, P. falciparum isolates were collected from patients with malaria aged between 6 months and 18 years in Kéniéroba, a village located in the SudanoGuinean area of Mali ( $12^{\circ} 6^{\prime} 50^{\prime \prime} \mathrm{N}$ and $\left.8^{\circ} 19^{\prime} 58^{\prime \prime} \mathrm{W}\right)$. Study participants were enrolled at the health center of Kéniéroba. The inclusion criterion was a $P$. falciparum mono-infection with a parasitemia between 1000 and 100,000 parasites per microliter. We collected $2-3 \mathrm{~mL}$ of venous blood samples on citric acid and a dextrose collection tube (ACD tubes) from each patient before treatment initiation, according to the NMCP's recommendations.

\subsection{Preparation of Anti-malarial Drugs}

Plasmodium falciparum isolates were tested using seven common anti-malarial drugs: CQ, QN, AQ, MQ, LUM, dihydroartemisinin (DHA), and PPQ. All drugs were obtained from Sigma. A stock solution of CQ diphosphate, $\mathrm{QN}, \mathrm{MQ}$, and DHA were prepared in 70\% ethanol. Amodiaquine and LUM were initially dissolved in methanol while PPQ was dissolved in lactic acid $0.5 \%$ first and then in dimethyl sulfoxide. Two-fold serial dilutions were prepared using sterile distilled water and distributed in duplicate into 96-well flat-bottom plates. Final concentration ranges of $2.44-2500 \mathrm{nM}$ for $\mathrm{CQ}, 4.88-5000 \mathrm{nM}$ for $\mathrm{QN}$, $1.22-1250 \mathrm{nM}$ for MQ, $1.22-1250 \mathrm{nM}$ for AQ, $0.34-350 \mathrm{nM}$ for LUM, $0.10-100 \mathrm{nM}$ for DHA, and $0.98-1000 \mathrm{nM}$ for PPQ. Fifty microliters of each diluted anti-malarial drug were added in a 96-well plate in duplicates. The 96-well plate was first dried up in the ambient air, then maintained at $4{ }^{\circ} \mathrm{C}$, and finally prepared for the culture within 2 weeks. The suitability of the prepared 96-well plate for in-vitro testing was continuously monitored using reference strain 3D7 $[13,14]$ during the test.

\subsection{Culture of the P. falciparum Isolates}

Briefly, 2-3 $\mathrm{mL}$ of whole blood was obtained by venipuncture from each patient with $P$. falciparum mono-infection and transported to the laboratory in Bamako, which is located within $2 \mathrm{~h}$ of our study site in Kenieroba. The plasma was removed after centrifugation of the whole blood. The cell pellet was washed three times with incomplete RPMI 1640 medium (Gibco ${ }^{\mathrm{TM}}$; Invitrogen Corporation, Carlsbad, California, USA) buffered with $25 \mathrm{mM}$ of HEPES (5.95 g/L) followed by a centrifugation at $2000 \mathrm{rpm}$ for $5 \mathrm{~min}$. Parasites were tested directly without culture adaptation. The suspension of parasites was distributed in 96-well plates preloaded with antimalarial drugs (as described above). Culture plates were incubated at $37{ }^{\circ} \mathrm{C}$ and $5 \% \mathrm{CO}_{2}$ for $72 \mathrm{~h}$. After this incubation (which corresponds to the schizont stage), a blood smear was prepared to confirm healthy growth of controls (drug-free parasites). Samples were then stored at $-20{ }^{\circ} \mathrm{C}$ overnight.

\subsection{Malaria SYBR ${ }^{\circledast}$ Green I-Based Fluorescence Assay}

The Malaria SYBR ${ }^{\circledR}$ Green I-Based Fluorescence Assay was performed as described in the modified version of the Smilkstein and colleagues paper [15] by Johnson et al. [16]. The plate was thawed for $1-2 \mathrm{~h}$ at room temperature to lyse the cells. Then, $100 \mu \mathrm{L}$ of $0.002 \% \mathrm{SYBR}^{\circledR}$ Green lysis buffer ( $10 \mathrm{~mL}$ of lysis buffer plus $2 \mu \mathrm{L}$ of $\mathrm{SYBR}^{\circledR}$ Green) was added in each well. The plate was then covered with aluminum foil and kept under agitation using a plate shaker at room temperature for $30 \mathrm{~min}$. The parasite growth was determined by measuring fluorescence of the SYBR ${ }^{\circledR}$ Green incorporated into nucleic acids of the parasites. The plate was read using a fluorometer plate reader using a $485-\mathrm{nm}$ excitation filter and a 538-nm emission filter. The $\mathrm{IC}_{50}$, defined as a drug concentration at which the $\mathrm{SYBR}^{\circledR}$ Green 
signal was $50 \%$ of that measured in drug-free control wells, was calculated by using the In-Vitro Analysis and Reporting Tool (IVART) software.

The $\mathrm{IC}_{50}$ threshold value measuring the parasite's sensitivity to a given drug was defined as reported in previous studies. These values were set at $100 \mathrm{nM}$ for the CQ $[17,18]$, $30 \mathrm{nM}$ for MQ [17-19], $80 \mathrm{nM}$ for AQ [18, 20], $150 \mathrm{nM}$ for LUM [18, 19, 21], $800 \mathrm{nM}$ for QN [18-20], $12 \mathrm{nM}$ for DHA [18], and 135 for PPQ [22, 23]. The SYBR ${ }^{\circledR}$ Green I-based method for determination of drug sensitivity concentrations provides results that are similar to other traditional methods, suggesting that this method can be routinely used to conduct surveillance for drug resistance with fresh or cultured parasites [24]. For example, Smilkstein and co-workers did not find a difference between the $\mathrm{IC}_{50}$ of standard anti-malarial drugs determined by the mean of a radio-isotopic approach from those generated using the SYBR ${ }^{\circledR}$ Green method [25].

\subsection{Statistical Analysis}

We analyzed results from samples that had good ex-vivo growth, free of contamination during culture, and with a good fit on the log dose-response curve. The concentration of a given anti-malarial drug that can inhibit the growth of $50 \%\left(\mathrm{IC}_{50}\right)$ of the parasites in culture was estimated from a dose-response curve by non-linear regression analysis using an online program previously described elsewhere [26, 27]. The program generated $\mathrm{IC}_{50}$ estimates with associated $95 \%$ confidence intervals (CIs). Estimated values with insufficient precision based on the CI were discarded. Geometric mean $\mathrm{IC}_{50}$ was calculated for each drug. Cross-susceptibility was analyzed using the Pearson correlation $(r)$ generated by the software GraphPad Prism version 8. A two-sided $p$ value $\leq 0.05$ was set as the significance threshold. The strength of correlation (with a significant linear relationship) was classified as moderate, if " $0.3<r<0.7$ " or strong, if " $r \geq 0.7$ ".

\section{Results}

A total of 100 parasite isolates were prepared for analysis. The $\mathrm{IC}_{50}$ was successfully determined for 74 (74\%) isolates for CQ, $74(74 \%)$ for QN, $73(73 \%)$ for $\mathrm{AQ}, 75(75 \%)$ for MQ, 73 (73\%) for LUM, 69 (69\%) for DHA, and 73 (73\%) for PPQ (Table 1). The geometric mean of the $\mathrm{IC}_{50}$ was $46.07 \mathrm{nM}$ with a 95\% CI range between 4.0 and 724.3 for CQ. Similarly, it was $264.97 \mathrm{nM}$ (39.90-1567.38) for QN, 25.5 nM (5.73-88) for AQ, $13.13 \mathrm{nM}$ (1.82-36.16) for MQ, $10.14 \mathrm{nM}(0.82-50.82)$ for LUM, $0.87 \mathrm{nM}$ (0.34-3.03) for DHA, and $15.86 \mathrm{nM}$ (3.72-35.72) for PPQ (Table 1). The proportion of resistant $P$. falciparum
Table 1 Geometric mean values of 50\% inhibitory concentration $\left(\mathrm{IC}_{50}\right)$ of different anti-malarial drugs

\begin{tabular}{llc}
\hline Molecules & $\begin{array}{l}\text { Number of } \\
\text { experiments }\end{array}$ & $\mathrm{IC}_{50}$ geometric mean $(95 \% \mathrm{CI})$ \\
\hline CQ & 74 & $46.07(4.90-724.3)$ \\
QN & 74 & $264.97(39.90-1567.38)$ \\
AQ & 73 & $25.5(5.73-88)$ \\
MQ & 75 & $13.13(1.82-36.16)$ \\
LUM & 73 & $10.14(0.82-50.82)$ \\
DHA & 69 & $0.87(0.34-3.03)$ \\
PPQ & 73 & $15.86(3.72-35.72)$ \\
\hline
\end{tabular}

$A Q$ amodiaquine, $C I$ confidence interval, $C Q$ chloroquine, $D H A$ dihydroartemisinin, $L U M$ lumefantrine, $M Q$ mefloquine, $P P Q$ piperaquine, $Q N$ quinine

Table 2 Ex-vivo response of isolates of Plasmodium falciparum from Kenieroba to antimalarial drugs

\begin{tabular}{lrrrlll}
\hline Drugs & $\begin{array}{l}\text { Cut-off values } \\
(\mathrm{nM})\end{array}$ & \multicolumn{2}{l}{ Resistant isolates } & & \multicolumn{2}{l}{$\begin{array}{l}\text { Sensitive } \\
\text { isolates }\end{array}$} \\
\cline { 3 - 4 } \cline { 6 - 7 } & & $N$ & $\%$ & & $N$ & $\%$ \\
\hline CQ & 100 & 9 & 12.2 & & 65 & 87.8 \\
AQ & 80 & 2 & 2.8 & & 71 & 97.2 \\
MQ & 30 & 1 & 1.3 & & 74 & 98.7 \\
QN & 800 & 19 & 25.7 & & 55 & 74.3 \\
LUM & 150 & 0 & 0 & & 73 & 100 \\
PPQ & 135 & 0 & 0 & & 73 & 100 \\
DHA & 12 & 0 & 0 & 69 & 100 \\
\hline
\end{tabular}

$A Q$ amodiaquine, $C Q$ chloroquine, $D H A$ dihydroartemisinin, $L U M$ lumefantrine, $M Q$ mefloquine, $P P Q$ piperaquine, $Q N$ quinine

isolates, as defined in the methods section, were $12.2 \%$ (9/74) for CQ, $25.7 \%$ (19/74) for QN, 2.7\% (2/73) for AQ, and $1.3 \%$ (1/75) for MQ. No resistant $P$. falciparum isolates to LUM, DHA, and PPQ were found in this study (Table 2).

A strong positive correlation was observed between the $\mathrm{IC}_{50}$ values for QN and AQ $\left(r=0.80 ; r^{2}=0.64, p<0.0001\right)$, suggesting an ex-vivo cross-resistance among these drugs. Moderate positive correlations were found between the $\mathrm{IC}_{50}$ values of CQ vs AQ $(r=0.57, p<0.0001), \mathrm{CQ}$ vs QN $(r=0.49, p<0.0001)$, DHA vs MFQ $(r=0.35, p=0.002)$, LUM vs MQ $(r=0.5, p<0.0001)$, DHA vs PPQ $(r=0.43$, $p<0.0001)$, and MQ vs PPQ $(r=0.45, p<0.0001)$. A weak positive correlation was observed between CQ vs MQ $\left(r=0.29 ; r^{2}=0.08, p=0.008\right)$ and DHA vs LUM $(r=0.30, p=0.012)$ (Table 3$)$. In contrast, negative correlations were observed between $\mathrm{IC}_{50}$ values of DHA vs QN ( $r=-0.35, p=0.002)$, LUM vs QN ( $r=-0.36$, 
Table 3 Correlation between anti-malarial drug 50\% maximal inhibitory concentration values

\begin{tabular}{llcc}
\hline Drug pairing & $\begin{array}{l}\text { Number of } \\
\text { experiments }\end{array}$ & $\begin{array}{l}\text { Correlation coef- } \\
\text { ficient }(r)\end{array}$ & $p$ value \\
\hline AQ vs CQ & $\mathbf{7 4}$ & $\mathbf{0 . 5 1 7}$ & $<\mathbf{0 . 0 0 1}$ \\
AQ vs DHA & $\mathbf{6 8}$ & $-\mathbf{0 . 2 8}$ & $\mathbf{0 . 0 1}$ \\
AQ vs LUM & $\mathbf{7 3}$ & $-\mathbf{0 . 4 5}$ & $<\mathbf{0 . 0 0 1}$ \\
AQ vs MQ & 74 & -0.04 & 0.72 \\
AQ vs PPQ & 74 & 0.07 & 0.55 \\
AQ vs QN & $\mathbf{7 3}$ & $\mathbf{0 . 8 0}$ & $<\mathbf{0 . 0 0 1}$ \\
CQ vs DHA & 70 & 0.096 & 0.41 \\
CQ vs LUM & 73 & 0.036 & 0.76 \\
CQ vs MQ & $\mathbf{7 6}$ & $\mathbf{0 . 2 9}$ & $\mathbf{0 . 0 0 8}$ \\
CQ vs PPQ & 75 & 0.21 & 0.06 \\
CQ vs QN & $\mathbf{7 3}$ & $\mathbf{0 . 4 9}$ & $<\mathbf{0 . 0 0 1}$ \\
DHA vs LUM & $\mathbf{6 7}$ & $\mathbf{0 . 3 0}$ & $\mathbf{0 . 0 1}$ \\
DHA vs MQ & 70 & 0.35 & 0.002 \\
DHA vs PPQ & $\mathbf{6 8}$ & $\mathbf{0 . 4 3}$ & $<\mathbf{0 . 0 0 0 1}$ \\
DHA vs QN & $\mathbf{6 7}$ & $\mathbf{0 . 3 5}$ & $\mathbf{0 . 0 0 2}$ \\
LUM vs MQ & $\mathbf{7 4}$ & $\mathbf{0 . 5}$ & $<\mathbf{0 . 0 0 1}$ \\
LUM vs PPQ & 73 & 0.11 & 0.34 \\
LUM vs QN & $\mathbf{7 1}$ & $\mathbf{0 . 3 6}$ & $\mathbf{0 . 0 0 2}$ \\
MQ vs PPQ & $\mathbf{7 5}$ & $\mathbf{0 . 4 5}$ & $<\mathbf{0 . 0 0 1}$ \\
MQ vs QN & 73 & -0.02 & 0.87 \\
PPQ vs QN & 72 & $\mathbf{- 0 . 1 0}$ & 0.37 \\
\hline
\end{tabular}

Significant correlation values are highlighted in bold

$A Q$ amodiaquine, $C Q$ chloroquine, $D H A$ dihydroartemisinin, $L U M$ lumefantrine, $M Q$ mefloquine, $P P Q$ piperaquine, $Q N$ quinine

$p=0.002)$, DHA vs AQ $(r=-0.28, p=0.01)$, and AQ vs LUM $(r=-0.45, p<0.0001)$.

\section{Discussion}

Ex-vivo assessment of the susceptibility of malaria parasites to antimalarial drugs remains an important component in the surveillance of the efficacy of anti-malarial drugs. As this method is largely independent on clinical factors, it provides information that complements clinical assessment of drug efficacy. The SYBR ${ }^{\circledR}$ Green method for assessing the outcome of the ex-vivo drug sensitivity test was revalidated and used to assess the responses of $P$. falciparum clinical isolates to seven anti-malarial drugs in Mali. To the best of our knowledge, this is the first study reporting the use of SYBR ${ }^{\circledR}$ Green method in Mali. We confirmed that this method is easy to use, reliable, and cheaper, as evidenced by the quick and successful evaluation of ex-vivo sensitivity $P$. falciparum isolates from Kéniéroba to a comprehensive panel of anti-malarial drugs, namely $\mathrm{CQ}, \mathrm{QN}, \mathrm{AQ}, \mathrm{MQ}$, LUM, DHA, and PPQ.
The prevalence of $P$. falciparum parasites with reduced susceptibility to QN was $25.7 \%$ in Kéniéroba. This prevalence was higher than those reported in Ghana, in 2011 (19.4\% [6/31]) [28] and Senegal in 2017 (9.7\% [3/31]) [29]. Globally, similar sensitivity of $P$. falciparum isolates to QN were found (mean $\mathrm{IC}_{50}=264.97 \mathrm{nM}$; $95 \% \mathrm{CI}$ 39.90-1567.38) as in a study conducted in Cote d'Ivoire in 2008 (mean $\mathrm{IC}_{50}=272.12 \mathrm{nM}$ ) by Touré et al. [30]. In contrast, a higher $\mathrm{IC}_{50}$ mean value for $\mathrm{QN}(355.37 \mathrm{nM})$ was reported by Kwansa-Bentum et al. in 2011 in Ghana [28]. To treat complicated malaria, QN by the intravenous route still remains the third-line treatment in Mali. Oral QN is also a recommended drug to treat uncomplicated malaria during the first trimester of pregnancy in Mali. As a reduced susceptibility in vitro is an early warning of impeding resistance, a very close follow-up of the resistance to $\mathrm{QN}$ is necessary in Mali.

In the present study, $12.2 \%$ of the P. falciparum isolates exhibited a reduced susceptibility to CQ $\left(\mathrm{IC}_{50} \geq 100 \mathrm{nM}\right.$ ). In 2004-6, a higher proportion of CQ-resistant P. falciparum isolates (60-69\%) was found in Mali [31]; however, we do not know if current circulating P. falciparum isolates are now sensitive to CQ because of the reduced selective pressure on that drug after the introduction of ACT in SubSaharan Africa. Reports from Malawi and Kenya suggest that likelihood [32-34]. This trend was also found in studies conducted by French scientists, using samples from patients with malaria from Senegal, Mali, Ivory Coast, and Cameroon $[35,36]$. The data indicate that it may be possible to re-introduce CQ as the first-line treatment of malaria in Mali because CQ is easily manufactured locally at a very low cost. Hence, we recommend routine monitoring of CQ resistance despite the widespread use of ACT.

Only $2.7 \%$ of the parasite isolates exhibited a reduced ex-vivo susceptibility to $\mathrm{AQ}\left(\mathrm{IC}_{50} \geq 80 \mathrm{nM}\right)$. This was interesting because $\mathrm{AQ}$ is one of the most commonly associated molecules with artemisinin in the ACT used in Mali. Higher prevalence of AQ-resistant parasites have been reported in 2017 in Senegal (28.1\%) [29] and in India (8\%) [37]. A strict adhesion to the NMCP's malaria treatment guideline in conjunction with the World Health Organization guidelines [38] may considerably reduce the spread of the AQ resistance in Africa.

In our study, $P$. falciparum isolates displayed a very high sensitivity to MQ, LUM, DHA, and PPQ similar to previous findings in Mali [39] and in Senegal [40]. To determine the most suitable antimalarial therapeutic combinations and to prevent the emergence of resistant parasites in the study site, correlations between $\mathrm{IC}_{50}$ values of the $P$. falciparum isolate were assessed. A positive correlation was found between the $\mathrm{IC}_{50}$ values for: $\mathrm{CQ}$ vs $\mathrm{AQ}, \mathrm{CQ}$ vs $\mathrm{QN}$, and $\mathrm{CQ}$ vs $\mathrm{MQ}$. The variation in the ex-vivo response to AQ (32\%), QN (24\%), and MQ (8\%) could be explained by the variation in 
response to CQ. However, the values of correlation coefficient remain low with no possible threat of a cross-resistance between CQ and AQ, CQ and QN, or CQ and MQ.

The artemisinin derivatives are recommended to be associated with LUM, AQ, PPQ, and MQ. In this study, we demonstrated a positive correlation between DHA vs LUM, DHA vs PPQ, and DHA vs MQ. Twelve percent, 9\%, and $18 \%$ of the variation in the ex-vivo response to MQ, LUM, and PPQ could be explained by variations in response to DHA. The values of the coefficient of determination between DHA vs MQ, DHA vs LUM, and DHA vs PPQ were too low to explain the cross-resistance. A cross-resistance between LUM and artemisinin derivatives has been observed previously [41]. There was a significantly positive correlation between the ex-vivo activity of MQ and PPQ $(r=0.45$; $\left.r^{2}=0.20, p<0.0001\right)$. A significant association between MQ and PPQ was already estimated with parasite isolates from Africa $(r=0.45)$ [42]. The correlation between MQ and PPQ might be partly explained by their similar mode of action. However, the values of the coefficient of determination remain low to fear cross-resistance between MQ and PPQ. A strong significant positive correlation was observed between QN and AQ $\left(r=0.80 ; r^{2}=0.64, p<0.0001\right)$. Sixtyfour percent $(64 \%)$ of the variation in the ex-vivo response to $\mathrm{QN}$ could be explained by variations in response to $\mathrm{AQ}$. The high value of the correlation coefficient raises a concern about a potential threat of cross-resistance between $\mathrm{QN}$ and AQ. From these data, we suggest that common mechanisms of action of these two drugs may explain the positive correlation between them, suggesting that parasites that have reduced susceptibility to QN would also be less susceptible to $\mathrm{AQ}$.

Cross-resistances between CQ-AQ, CQ-QN, and QN-AQ have already been reported in previous studies [43-45]. Cross-resistance is thought to occur when a drug confers resistance to other drugs that have a similar mode of action or belong to the same chemical group. Cross-resistance affects essentially drugs belonging to the same chemical family or drugs with similar modes of action or modes of transportation [46-49]. Theoretically, parasites are more prone to be sensitive to a molecule whose $\mathrm{IC}_{50}$ is inversely proportional to that the one of a molecule the parasites display resistance. Data from our study revealed light to moderate, but significant negative correlations between sensitivities of $P$. falciparum isolates to AQ and DHA $(r=-0.28$, $p=0.01)$, DHA and QN $(r=-0.35, p=0.01)$, and AQ and LUM $(r=-0.45, p<0.001)$. The negative correlation observed between DHA and AQ implies that the use of artemisinin in combination with $\mathrm{AQ}$ is very relevant in Mali. Our findings are reassuring considering the emergence of resistance to artemisinin derivatives [50-53].

\section{Conclusions}

Plasmodium falciparum isolates were highly sensitive to DHA, LUM, and PPQ. In addition, few isolates showed a reduced sensitivity to AQ and MQ (only one isolate). A much lower sensitivity to QN was also detected. Our data confirm an increasing trend in the sensitivity of the P. falciparum field isolate to CQ in Mali. This study provides valuable data that may considerably contribute to a better clinical management of patients with malaria and guide the decision-making process for an effective national strategy for malaria treatment.

Acknowledgements We thank the parents, guardians, and children who participated in this study, and the technical, clinical, and nursing staff for assistance. We are grateful to many colleagues at the Malaria Research and Training Center for providing critical reviews of the manuscript that have helped improve the content.

Author Contributions KT, SASD, and MD provided the study design. KT, SASD, IS, DSK, BK, and MD provided the sample and collected the data. KT and SASD performed the data analysis. KT wrote the manuscript. KT, SASD, DD, SD, MS, SB, MAG, SD, and MD edited and reviewed the manuscript.

Data Availability All data generated or analyzed during this study are included in the published article.

\section{Compliance with Ethical Standards}

Funding This study is supported by the University of Sciences, Techniques, and Technologies of Bamako (USTTB).

Conflict of interest Karim Traoré, Seidina A.S. Diakité, Sekou Bah, Drissa S. Konaté, Djeneba Dabitao, Ibrahim Sanogo, Modibo Sangaré, Souleymane Dama, Bourama Keita, Mory Doumbouya, Merepen A. Guindo, Seydou Doumbia, and Mahamadou Diakité have no conflicts of interest that are directly relevant to the content of this article.

Ethics approval The study was approved by the Ethics Committee of the Faculty of Medicine and Pharmacy of the University of Sciences, Techniques, and Technologies of Bamako (USTTB), Mali.

Consent to participate All study participants signed a written consent or assent (for children) form to participate in this study.

Consent for publication All authors read and approved the final manuscript.

Open Access This article is licensed under a Creative Commons Attribution-NonCommercial 4.0 International License, which permits any non-commercial use, sharing, adaptation, distribution and reproduction in any medium or format, as long as you give appropriate credit to the original author(s) and the source, provide a link to the Creative Commons licence, and indicate if changes were made. The images or other third party material in this article are included in the article's Creative Commons licence, unless indicated otherwise in a credit line to the material. If material is not included in the article's Creative Commons licence and your intended use is not permitted by statutory regulation or exceeds the permitted use, you will need to obtain permission 
directly from the copyright holder. To view a copy of this licence, visit http://creativecommons.org/licenses/by-nc/4.0/.

\section{References}

1. World Health Organization. World malaria report 2015. Geneva: World Health Organization; 2015.

2. Packard RM. The origins of antimalarial-drug resistance. N Engl J Med. 2014;371(5):397-9.

3. Wongsrichanalai $\mathrm{C}$, et al. Epidemiology of drug-resistant malaria. Lancet Infect Dis. 2002;2(4):209-18.

4. Spencer HC. Drug-resistant malaria: changing patterns mean difficult decisions. Trans R Soc Trop Med Hyg. 1985;79(6):748-58.

5. Wernsdorfer WH, Payne D. The dynamics of drug resistance in Plasmodium falciparum. Pharmacol Ther. 1991;50(1):95-121.

6. World Health Organization. Guidelines for the treatment of malaria. Geneva: World Health Organization; 2011.

7. Phyo AP, et al. Emergence of artemisinin-resistant malaria on the western border of Thailand: a longitudinal study. Lancet. 2012;379(9830):1960-6.

8. Amaratunga $\mathrm{C}$, et al. Artemisinin-resistant Plasmodium falciparum in Pursat province, western Cambodia: a parasite clearance rate study. Lancet Infect Dis. 2012;12(11):851-8.

9. Ariey F, et al. A molecular marker of artemisinin-resistant Plasmodium falciparum malaria. Nature. 2014;505(7481):50-5.

10. Tacoli $\mathrm{C}$, et al. Artemisinin resistance-associated K13 polymorphisms of Plasmodium falciparum in Southern Rwanda, 2010-2015. Am J Trop Med Hyg. 2016;95(5):1090-3.

11. Zhang J, et al. In vitro susceptibility of Plasmodium falciparum isolates from the China-Myanmar border area to artemisinins and correlation with K13 mutations. Int J Parasitol Drugs Drug Resist. 2019;10:20-7.

12. OMS. Rapport mondial sur l'efficacité des médicaments antipaludiques et la pharmacorésistance: 2000-2010. Genève, S.O.m.d.l.s., rapport mondial sur l'efficacité des médicaments antipaludiques et la pharmacorésistance: 2000-2010. Genève: Organisation mondiale de la santé; 2011.

13. Walliker D, et al. Genetic analysis of the human malaria parasite Plasmodium falciparum. Science. 1987;236(4809):661-6.

14. Wellems TE, et al. Chloroquine resistance not linked to mdr-like genes in a Plasmodium falciparum cross. Nature. 1990;345(6272):253-5.

15. Smilkstein M, et al. Simple and inexpensive fluorescence-based technique for high-throughput antimalarial drug screening. Antimicrob Agents Chemother. 2004;48(5):1803-6.

16. Johnson JD, et al. Assessment and continued validation of the malaria SYBR Green I-based fluorescence assay for use in malaria drug screening. Antimicrob Agents Chemother. 2007;51(6):1926-33.

17. Brasseur $\mathrm{P}$, et al. Emergence of mefloquine-resistant malaria in Africa without drug pressure. Lancet. 1990;336(8706):59.

18. Pradines B, et al. Reduced in vitro susceptibility to artemisinin derivatives associated with multi-resistance in a traveller returning from South-East Asia. Malar J. 2011;10:268.

19. Reynes JM, et al. In vitro responses of Plasmodium falciparum isolates to five antimalaria drugs in French Guiana during 1994 and 1995. Mem Inst Oswaldo Cruz. 1997;92(2):251-2.

20. Dedet JP, et al. In vitro activity of various antimalarials (chloroquine, amodiaquine, quinine and mefloquine) against 32 isolates of Plasmodium falciparum in French Guiana. Bull Soc Pathol Exot Filiales. 1988;81(1):88-93.

21. Mungthin M, et al. Association between the pfmdr1 gene and in vitro artemether and lumefantrine sensitivity in Thai isolates of Plasmodium falciparum. Am J Trop Med Hyg. 2010;83(5):1005-9.

22. Fall B, et al. Ex vivo susceptibility of Plasmodium falciparum isolates from Dakar, Senegal, to seven standard anti-malarial drugs. Malar J. 2011;10:310.

23. Pascual A, et al. Multinormal in vitro distribution of Plasmodium falciparum susceptibility to piperaquine and pyronaridine. Malar J. 2015;14:49.

24. Bacon DJ, et al. Comparison of a SYBR Green I-based assay with a histidine-rich protein II enzyme-linked immunosorbent assay for in vitro antimalarial drug efficacy testing and application to clinical isolates. Antimicrob Agents Chemother. 2007;51(4):1172-8.

25. Afari EA, et al. In vitro responses of $P$. falciparum parasites to chloroquine, amodiaquine and quinine in two ecological zones in Ghana. Cent Afr J Med. 1993;39(7):136-40.

26. Le Nagard $\mathrm{H}$, et al. Online analysis of in vitro resistance to antimalarial drugs through nonlinear regression. Comput Methods Progr Biomed. 2011;104(1):10-8.

27. Kaddouri $\mathrm{H}$, et al. Assessment of the drug susceptibility of Plasmodium falciparum clinical isolates from africa by using a Plasmodium lactate dehydrogenase immunodetection assay and an inhibitory maximum effect model for precise measurement of the 50-percent inhibitory concentration. Antimicrob Agents Chemother. 2006;50(10):3343-9.

28. Kwansa-Bentum B, et al. Plasmodium falciparum isolates from southern Ghana exhibit polymorphisms in the SERCA-type PfATPase6 though sensitive to artesunate in vitro. Malar J. 2011;10:187.

29. Diawara S, et al. Confirmation of Plasmodium falciparum in vitro resistance to monodesethylamodiaquine and chloroquine in Dakar, Senegal, in 2015. Malar J. 2017;16(1):118.

30. Touré AO, et al. In vitro susceptibility of $P$. falciparum isolates from Abidjan (Cote d'Ivoire) to quinine, artesunate and chloroquine. Sante. 2008;18(1):43-7.

31. Kaddouri $\mathrm{H}$, et al. Baseline in vitro efficacy of ACT component drugs on Plasmodium falciparum clinical isolates from Mali. Int J Parasitol. 2008;38(7):791-8.

32. Kublin JG, et al. Reemergence of chloroquine-sensitive Plasmodium falciparum malaria after cessation of chloroquine use in Malawi. J Infect Dis. 2003;187(12):1870-5.

33. Laufer MK, et al. Return of chloroquine antimalarial efficacy in Malawi. N Engl J Med. 2006;355(19):1959-66.

34. Mwai L, et al. Chloroquine resistance before and after its withdrawal in Kenya. Malar J. 2009;8:106.

35. Gharbi M, et al. Longitudinal study assessing the return of chloroquine susceptibility of Plasmodium falciparum in isolates from travellers returning from West and Central Africa, 2000-2011. Malar J. 2013;12:35.

36. Lu F, et al. Return of chloroquine sensitivity to Africa? Surveillance of African Plasmodium falciparum chloroquine resistance through malaria imported to China. Parasit Vectors. 2017;10(1):355

37. Sharma $\mathrm{S}$, et al. Correlation of in vitro sensitivity of chloroquine and other antimalarials with the partner drug resistance to Plasmodium falciparum malaria in selected sites of India. Indian J Med Microbiol. 2017;35(4):485-90.

38. World Health Organization. WHO briefing on malaria treatment guidelines and artemisinin monotherapies. Geneva: World Health Organization; 2006.

39. Dama S, et al. Reduced ex vivo susceptibility of Plasmodium falciparum after oral artemether-lumefantrine treatment in Mali. Malar J. 2017;16(1):59.

40. Fall B, et al. Plasmodium falciparum susceptibility to anti-malarial drugs in Dakar, Senegal, in 2010: an ex vivo and drug resistance molecular markers study. Malar J. 2013;12:107. 
41. Pradines B, et al. In vitro activities of benflumetol against 158 Senegalese isolates of Plasmodium falciparum in comparison with those of standard antimalarial drugs. Antimicrob Agents Chemother. 1999;43(2):418-20.

42. Robert MG, et al. Absence of a high level of duplication of the plasmepsin II gene in Africa. Antimicrob Agents Chemother. 2018;62(11):e00374-e418.

43. Ringwald $P$, et al. In vitro culture and drug sensitivity assay of Plasmodium falciparum with nonserum substitute and acute-phase sera. J Clin Microbiol. 1999;37(3):700-5.

44. Pradines B, et al. In-vitro activity of pyronaridine and amodiaquine against African isolates (Senegal) of Plasmodium falciparum in comparison with standard antimalarial agents. J Antimicrob Chemother. 1998;42(3):333-9.

45. Ouedraogo JB, et al. In vitro sensitivity of Plasmodium falciparum to halofantrine compared with chloroquine, quinine and mefloquine in the region of Bobo-Dioulasso, Burkina Faso (West Africa). Trop Med Int Health. 1998;3(5):381-4.

46. Ringwald P, Bickii J, Basco LK. In vitro activity of dihydroartemisinin against clinical isolates of Plasmodium falciparum in Yaounde, Cameroon. Am J Trop Med Hyg. 1999;61(2):187-92.

47. East African Network for Monitoring Antimalarial Treatment (EANMAT). Monitoring antimalarial drug resistance within
National Malaria Control Programmes: the EANMAT experience. Trop Med Int Health. 2001;6(11):891-8.

48. Randrianarivelojosia M, et al. In-vitro sensitivity of Plasmodium falciparum to chloroquine, halofantrine, mefloquine and quinine in Madagascar. East Afr Med J. 2002;79(5):237-41.

49. Yavo W, et al. In vitro susceptibility of Plasmodium falciparum to monodesethylamodiaquine, quinine, mefloquine and halofantrine in Abidjan (Cote d'Ivoire). Afr Health Sci. 2010;10(2):111-6.

50. Na-Bangchang $\mathrm{K}$, et al. Declining in efficacy of a three-day combination regimen of mefloquine-artesunate in a multi-drug resistance area along the Thai-Myanmar border. Malar J. 2010;9:273.

51. Woitsch B, et al. Susceptibility to chloroquine, mefloquine and artemisinin of Plasmodium vivax in northwestern Thailand. Wien Klin Wochenschr. 2007;119(19-20 Suppl. 3):76-82.

52. Noedl H, et al. Artemisinin resistance in Cambodia: a clinical trial designed to address an emerging problem in Southeast Asia. Clin Infect Dis. 2010;51(11):e82-e8989.

53. Spring MD, et al. Dihydroartemisinin-piperaquine failure associated with a triple mutant including kelch13 C580Y in Cambodia: an observational cohort study. Lancet Infect Dis. 2015;15(6):683-91. 